Check for updates

Cite this: RSC Adv., 2019, 9, 9117

Received 3rd December 2018

Accepted 13th March 2019

DOI: 10.1039/c8ra09952a

rsc.li/rsc-advances

\section{Titanium discs coated with 3,4-dihydroxy-L- phenylalanine promote osteogenic differentiation of human bone mesenchymal stem cells in vitro}

\author{
Ting Ma, DiD $\dagger^{\mathrm{a}}$ Xi-Yuan Ge, (D) $\dagger^{\mathrm{b}} \mathrm{Ke}-\mathrm{Yi} \mathrm{Hao}{ }^{\mathrm{a}}$ Xi Jiang, ${ }^{a}$ Yan Zheng, ${ }^{a}$ Ye Lin ${ }^{\star a}$ \\ and $Y u$ Zhang $(\mathbb{D}$ *a
}

\begin{abstract}
The bioinspired material 3,4-dihydroxy-L-phenylalanine (DOPA) is commonly used as a basic layer in surface modification for osteogenesis; however, its effects on bone remodeling and the underlying mechanisms remain unclear. Here, we investigated the effect of DOPA-coated surfaces on human bone marrow-derived mesenchymal stem cells in vitro. Cells cultured on DOPA-modified titanium discs exhibited enhanced cellular adhesion and spreading compared with cells on non-treated surfaces. Moreover, DOPA-coating promoted greater cell proliferation and osteogenic differentiation, as determined using cell counting kit-8 (CCK-8) assay, alkaline phosphatase activity test and quantitative mineralization measurements. Furthermore, microarray analysis revealed that genes participating in focal adhesion were upregulated on DOPA-coated surfaces. Our results indicate that the application of a simple DOPA coating can promote osteogenic differentiation of osteoprogenitor cells, improving new bone formation and bone remodeling around implantable devices in tissue engineering.
\end{abstract}

\section{Introduction}

Biomaterial surfaces can significantly influence cellular functions and promote osseointegration. The term osseointegration is used to describe the direct and functional connection between the surface of titanium and bone. ${ }^{1}$ Because of their excellent biocompatibility and biomechanical properties, pure titanium and its alloys have been widely applied in maxillofacial surgery and dental implants. However, fibrous tissue formation at the implant-bone interface can jeopardize the stability of these devices. ${ }^{2}$ Many chronic systemic metabolic diseases or local factors can induce poor bone conditions around the implant site and threaten long-term fixation. ${ }^{3}$ In these scenarios, a better understanding of the biological milieu of fixed orthopedic implants is needed to ensure enhanced bone remodeling. However, to date, studies have mainly focused on the potential advantages of improving osseointegration and

\footnotetext{
${ }^{a}$ Department of Oral Implantology, Peking University School and Hospital of Stomatology \& National Clinical Research Center for Oral Diseases \& National Engineering Laboratory for Digital and Material Technology of Stomatology \& Beijing Key Laboratory of Digital Stomatology, 22 Zhongguancun South Avenue, Haidian District, Beijing 100081, PR China. E-mail: yorcklin@263.net; zhang76yu@ 163.com

${ }^{b}$ Central Laboratory, Peking University School and Hospital of Stomatology \& National Clinical Research Center for Oral Diseases \& National Engineering Laboratory for Digital and Material Technology of Stomatology \& Beijing Key Laboratory of Digital Stomatology, 22 Zhongguancun South Avenue, Haidian District, Beijing 100081, PR China

$\dagger$ These authors contributed equally to this work.
}

bone remodeling around osseointegrated implants via local implant coating modifications. ${ }^{4}$

Positive and desirable interactions between stem cells and biomaterial interfaces can offer an alternative and promising therapeutic approach. Tissue regeneration relies on stem cell proliferation and differentiation. ${ }^{5}$ Studies have suggested that the osteogenic potential of implants is dependent on the first group of mesenchymal stem cells (MSCs) colonizing the implant surface and migrating through the peri-implant clot. ${ }^{6}$ Rather than contacting the implant surface directly, adherent cells contact the implant surface through proteins.' Therefore, surface topography and chemical composition of implants can impact protein adsorption, cellular attachment, proliferation and osteogenic differentiation. ${ }^{8}$ Moreover, cells colonized on the implant surface can also affect those distal to the implant surface by paracrine regulation. MSCs can differentiate into various types of cells, including osteocytes, adipocytes and chondrocytes. MSCs also have immunomodulatory properties, which can be exploited to guide therapeutic treatments for bone repair., ${ }^{9,10}$ Many matrix-mimicking biomaterials were first developed to favor the cellular behaviors of MSCs. However, recent advancements in bone tissue engineering include the development of ECM mimic silk fibroin, electroconductive natural polymer-based hydrogels (ENPHs), 3D printed porous ceramic scaffolds, and bioreactor systems for musculoskeletal tissue engineering. ${ }^{11-13}$ These bone tissue engineering scaffolds exhibit exciting properties, including connectivity (which could be used as a framework to guide cell and tissue growth to a certain form), carrier (which transports signaling molecules 
for osteoinduction), and cellular functions (by allowing cell adhesion through specific sites). ${ }^{\mathbf{1 4}}$ Therefore, to further modulate osseointegration, investigations into the underlying mechanisms of how MSCs behave on implant interfaces are urgently needed.

Inspired by the adhesive proteins secreted by mussels to attach onto wet surfaces, it was found that dopamine (DA) selfpolymerizes in aqueous solution to adhere onto solid surfaces, ${ }^{15}$ coating both inorganic and organic surfaces. To provide further modifications, surfaces can be coated with polydopamine (pDA) or 3,4-dihydroxy-L-phenylalanine (DOPA), forming a basic layer. Many researchers have developed in vitro linking approaches that bind to the DOPA or pDA layer with peptides, growth factors (nerve growth factor or angiogenic factor), or by immobilizing nano-hydroxyapatite particles to the substance, to modulate their biological effects. ${ }^{\text {16-20 }}$ The biochemistry of mussel adhesion in aqueous conditions is mainly dependent on Mytilus edulis foot protein-5 (Mefp-5), which contains $\sim 30$ mol\% DOPA motifs. Adhesion and cross-linking of mussel proteins correlate with the action of catechol groups in DOPA. ${ }^{21,22}$ Therefore, mussel-derived surface modifications can be further modified for biomedical applications. Surface modifications with DOPA are often combined with factors that promote bone remodeling for dental implant osseointegration, whereby adherence of undifferentiated MSCs to the implant site is a critical step in the process. Although DOPA coatings are readily used as linkers activated by amine or thiol groups, the use of DOPA-modified substances in manipulating cells, especially bone mesenchymal stem cells, remains unexplored. In this study, we first investigated the substrate properties of titanium discs before and after DOPA-coating. We next analyzed the effects of DOPA surface modifications on cell adhesion, proliferation, and differentiation of BM-MSCs in vitro. Finally, we analyzed the effects of DOPA-coating on the mRNA expression profiles of BM-MSCs.

\section{Methods}

\subsection{Titanium sample preparation and DOPA surface modification}

Commercial pure titanium discs ( $1 \mathrm{~mm}$ thick, $15 \mathrm{~mm}$ diameter) were sandblasted using $0.25-0.50 \mathrm{~mm} \mathrm{Al}_{2} \mathrm{O}_{3}$ particles and acidetched in $\mathrm{HCl} / \mathrm{H}_{2} \mathrm{SO}_{4}$ (sandblasted and acid-etched; SLA; Wego Jericom Biomaterials Co., Weihai, China). To create SLA-DOPA samples, discs were coated in $5 \mathrm{mg} \mathrm{mL}^{-1}$ DOPA solution (Sigma-Aldrich, St Louis, MO, USA) in $10 \mathrm{mM}$ Tris-HCl (pH 8.5) for $24 \mathrm{~h}$ at $37^{\circ} \mathrm{C}$, and the stain was washed with deionized water and sterilized before use.

\subsection{Characterization of titanium discs}

The surface morphologies of the DOPA-modified SLA titanium discs and SLA discs were observed by scanning electron microscopy (SEM; S-4800, Hitachi, Tokyo, Japan). Surface morphology and roughness were also examined by threedimensional (3D) optical microscopy (Contour GT; Bruker, Billerica, MA, US). A standard contact angle goniometer
(OCA20; Dataphysic, Filderstadt, Germany) with drop image software was used to characterize surface wettability using deionized water.

\subsection{Cell culture}

Human BM-MSCs (Cyagen Bioscience Inc., Sunnyvale, CA, USA) were cultured at $37^{\circ} \mathrm{C}$ in $\alpha$-Minimal Essential Medium ( $\alpha$-MEM; Gibco-Life Technologies, Grand Island, NY, USA) containing $10 \%$ fetal bovine serum (FBS; Gibco) and 1\% penicillin/ streptomycin in a humidified atmosphere containing $5 \% \mathrm{CO}_{2}$. Cells were passaged $1: 3$ every 2-3 days after reaching 70-90\% confluence. Osteogenic media ( $\alpha$-MEM, 10\% FBS, $10 \mathrm{mM}$ glycerophosphate, $50 \mu \mathrm{g} \mathrm{mL} \mathrm{m}^{-1} \mathrm{~L}$-ascorbic acid 2-phosphate, $100 \mathrm{nM}$ dexamethasone) was replaced every 3 days. Passages 4-7 were used for in vitro studies.

\subsection{Measurements of cell adhesion}

BM-MSCs $\left(2 \times 10^{3}\right.$ cells per $\left.\mathrm{mL}\right)$ were seeded onto SLA or SLADOPA titanium discs in 24-well plates with proliferation medium ( $\alpha$-MEM; containing $10 \%$ fetal bovine serum and $1 \%$ penicillin/streptomycin). These were cultured for 1,3 or $24 \mathrm{~h}$, fixed in $4 \%$ paraformaldehyde solution for $10 \mathrm{~min}$, permeabilized in $0.1 \%$ Triton $\mathrm{X}-100 /$ phosphate buffered saline (PBS), and incubated in $200 \mu \mathrm{L}$ of $1 \mu \mathrm{g} \mathrm{mL} \mathrm{mb}^{-1}$ phalloidin-FITC (Sigma, P5282) in PBS for $1 \mathrm{~h}$ to label the actin cytoskeleton. The nuclei were labeled with 4 $^{\prime}$,6-diamidino-2-phenylindole dihydrochloride (DAPI; Cell Signaling Technology, Danvers, MA, USA). Samples were examined by confocal laser scanning microscopy (CLSM, Carl Zeiss, Jena, Germany) at $488 \mathrm{~nm}$ wavelength or under UV light. To quantify cell adhesion, BMMSCs $\left(6 \times 10^{3}\right.$ cells per $\left.\mathrm{mL}\right)$ were seeded onto titanium discs, cultured for 1, 3 or $24 \mathrm{~h}$, washed in PBS three times to remove non-adherent cells, fixed, permeabilized, and nuclei were labeled using DAPI. Cell numbers in three randomly selected light microscope fields (Olympus, Tokyo, Japan; $\times 10$ objective) were calculated using Image-Pro Plus 6.0 software (Media Cybernetics, Silver Spring, Maryland, USA).

\subsection{Cell proliferation assay}

To measure cell proliferation, BM-MSCs were cultured on SLA and SLA-DOPA titanium discs for 1, 3 or 7 days in proliferation medium. At each time point, three samples from each group were analyzed using the Cell Counting Kit-8 (CCK-8, Dojindo, Kumamoto, Japan). Optical density (OD) was measured at 450 nm using ELX-808 Absorbance Microplate Recorder (BioTek, Winooski, VT, USA).

\subsection{Alkaline phosphatase (ALP) activity}

To assay alkaline phosphatase (ALP), BM-MSCs $\left(6 \times 10^{3}\right.$ cells per well) were seeded onto SLA or SLA-DOPA titanium discs in 24-well culture plates in osteogenic medium. After 7 days, samples were rinsed three times in PBS and cells were lysed in $0.1 \%$ Triton X-100. ALP activity was measured using an ALP kit (Jian cheng Bioengineering Institute, Nanjing, China) and 
normalized to total protein. All experiments were performed in triplicate.

\subsection{Quantitative calcium deposition assay}

To assay mineralization, MSCs (a density of $5 \times 10^{3}$ cells per well) were seeded on SLA or SLA-DOPA titanium discs. After $24 \mathrm{~h}$, the proliferation medium ( $\alpha$-MEM; containing $10 \%$ fetal bovine serum and $1 \%$ penicillin/streptomycin) was replaced with osteogenic differentiation medium and cells were incubated for 21 days. At the time point, samples were washed twice in PBS (pH 7.4). Cells were then fixed in $4 \%$ paraformaldehyde, stained with $2 \%(\mathrm{w} / \mathrm{v})$ Alizarin Red S solution ( $\mathrm{pH} 4.2$; SigmaAldrich) and incubated for $10 \mathrm{~min}$, washed three times with distilled water, incubated in 10\% 1-hexadecylpyridinium chloride, and absorbance of the samples was quantified at $540 \mathrm{~nm}$ using a microplate reader.

\subsection{Microarray analysis}

BM-MSCs were seeded onto SLA or SLA-DOPA titanium discs ( $n$ $=3$ ), cultured for 7 days, total RNA was extracted from cells using TRIzol reagent (Invitrogen Life Technologies, Carlsbad, CA, USA). We conducted gene expression profiling of BM-MSCs using Affymetrix Human U133 plus 2.0 arrays (Affymetrix, Santa Clara, CA). Gene ontology (GO; http://www.geneontology.org) analysis was performed using software from Shanghai Biotechnology Corporation. Microarrays were performed by Shanghai Biotechnology Corporation.

\subsection{Quantitative real-time PCR assay}

Total mRNA was isolated from cells seeded on SLA and SLADOPA at day 7 using Trizol reagent. cDNA was synthesized by using a Reverse Transcription kit (Promega, Madison, WI, USA). Fast Start Universal SYBR Green Master (ROX; Roche, Indianapolis, IN, USA) was used, and an ABI 7500 Real-Time PCR system (Applied Biosystems, Foster City, CA, USA) was applied in the present study. Relative quantification was performed using the ${ }^{{ }^{\Delta}} C_{\mathrm{t}}$ method. The sequences of primers used for syndecan-1 (SDC1), dipeptidyl peptidase 4 (DPP4), and neurofibromin 2 (NF2) are listed in Table 1.

\subsection{Statistical analysis}

All data are mean \pm standard deviation (SD). The $t$-test was used to evaluate differences between groups with SPSS 17.0 (SPSS Inc., Chicago, USA) software for Microsoft Windows; $P<0.05$ was considered significant.

\section{Results and discussion}

Titanium implants are widely used in dentistry and orthopedics, owing to their excellent biocompatibility and mechanical properties. A previous study found that while the thin native oxide film on titanium implants exhibited great biocompatibility, it was considered inert as it rarely bonded to the surrounding tissue. ${ }^{23}$ To enhance titanium biocompatibility and shorten the duration of bone-healing, various methods of surface modification have been employed that alter the physical, chemical and biological properties of implants. ${ }^{24}$ Particularly, the application of a simple DOPA coating film via oxidative polymerization has provided a controlled and versatile approach for surface modification. ${ }^{25}$ DOPA modification is an economical and controlled process. It can be used to modify subjects with complex geometry and in conjugation of other biomolecules as a platform for further modifications. ${ }^{26}$

\subsection{Effect of DOPA-coating on the surface properties of sand-blasted and acid-etched (SLA) titanium}

Fig. 1a shows the surface morphologies of SLA and SLA-DOPA titanium discs as observed by SEM. The partial aggregates of polymerized DOPA seen on the SLA-DOPA discs confirm successful formation of a DOPA film coating the surfaces. Dopamine particles measuring from 10-100 nm wide and 10$25 \mathrm{~nm}$ high can occur on polished surfaces. ${ }^{27,28}$ Bone tissue is composed of complex three dimensional hierarchical structures, which contribute to the integral mechanical properties of bone. Therefore, nanomaterials with macro- and microstructures that can mimic bone tissue prove promising candidates in the development of bone engineering scaffolds. ${ }^{29}$ Our $3 \mathrm{D}$ optical microscopy analysis revealed that SLA implants produced a microroughened surface topography with large cavities. We also found that modification using simple DOPA increased the average surface roughness from $1.742 \pm 0.173 \mu \mathrm{m}$ (for SLA alone) to $2.283 \pm 0.166 \mu \mathrm{m}$ (SLA-DOPA; $P<0.05$ ). These results indicate that when titanium discs are immersed in DOPA solution, self-polymerization particles or layers spontaneously grow on the titanium surface. Moreover, after observing the micro-scale structure of the titanium surface, we observed the surface roughness increased slightly between the DOPAmodified surface and the control group. However, the underlying surface topography did not significantly change.

The water contact angle (CA) of a material's surface, which is the angle between the liquid drop (tangent) and the solid surface (horizontal), reflects its hydrophilicity; $<90^{\circ}$ is considered hydrophilic, while $>90^{\circ}$ is considered hydrophobic. We

Table 1 Primers used for quantitative real-time PCR

\begin{tabular}{lll}
\hline Gene & Forward sequence $\left(5^{\prime}-3^{\prime}\right)$ & Reverse sequence $\left(5^{\prime}-3^{\prime}\right)$ \\
\hline GAPDH & $5^{\prime}$-GGCAAGTTCAACGGCACAGT-3' & $5^{\prime}$-GCCAGTAGACTCCACGACAT-3' \\
SDC1 & $5^{\prime}$-CTGCCGCAAATTGTGGCTAC-3' & $5^{\prime}$-TGAGCCGGAGAAGTTGTCAGA-3' \\
DPP4 & $5^{\prime}$-AGTGGCACGGCAACACATT-3 & $5^{\prime}$-AGAGCTTCTATCCCGATGACTT-3' \\
NF2 & $5^{\prime}$-AGTGGCCTGGCTCAAAATGG-3' & $5^{\prime}$-TGTTGTGTGATCTCCTGAACCA-3'
\end{tabular}


a
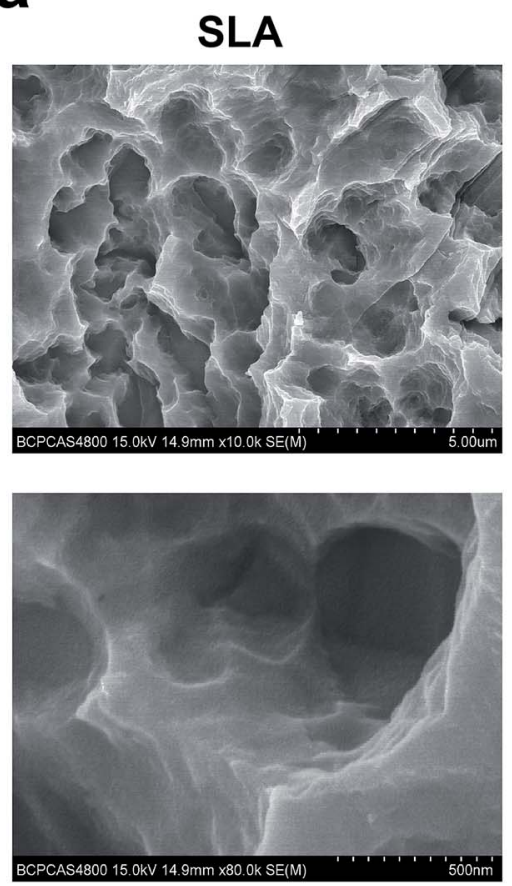

b
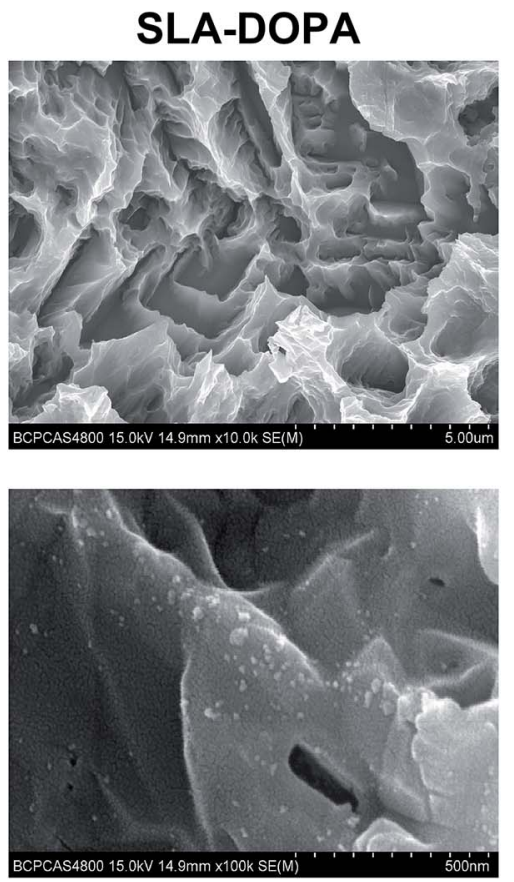

C
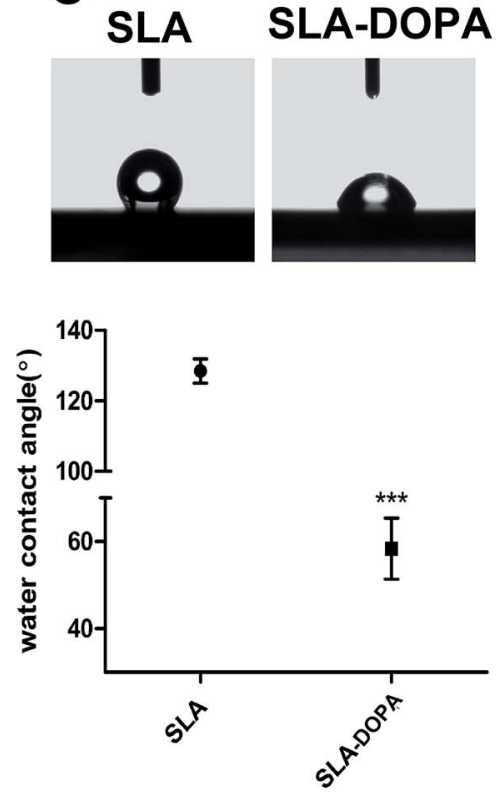

SLA

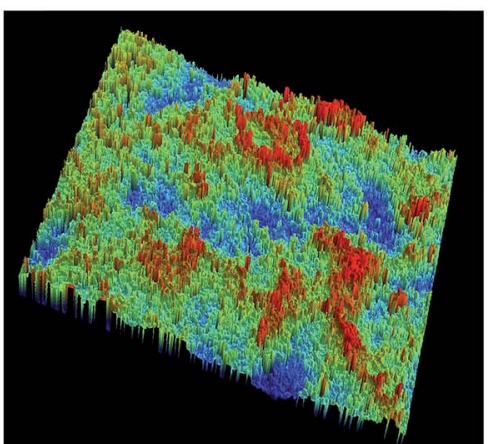

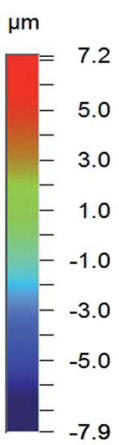

SLA-DOPA

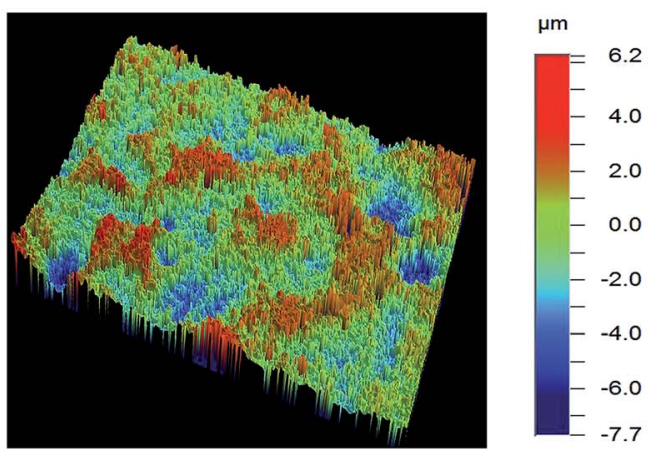

Fig. 1 Characterization of sandblasted and acid-etched (SLA) titanium and SLA-3,4-dihydroxy-L-phenylalanine (DOPA) titanium surfaces. (a) Scanning electron microscopy analysis shows partial aggregates of polymerized DOPA on SLA-DOPA surfaces. (b) Three-dimensional optical microscopy images. (c) Water contact angle: SLA-DOPA surface is more hydrophilic than the SLA surface. Data are mean \pm SD $(* * * P<0.001, n=3)$.

showed that DOPA coating significantly reduced the contact angle, from $128.41 \pm 6.83^{\circ}$ to $61.79 \pm 7.54^{\circ}$ (Fig. 1c). Surface energy can be measured indirectly using the liquid-solid CA. There are many methods to calculate the surface free energy, such as Owens-Wendt-Rabel-Kaelble (ORWK) method, Zisman approach, and Good-Girifalco method et al. However, serious deviations in surface free energy have been reported using different approaches. ${ }^{30}$ Meanwhile, calculation of CA hysteresis of surface biomaterial adhesion strengths can provide better insight into the bioadhesion mechanism. ${ }^{31}$ Albeit, the values of CAs of clinical dental implants range widely; it is generally accepted that surface hydrophilicity is a critical factor for protein and cell adhesion during early stages of osseointegration. ${ }^{32}$ For example, chemical modification of hydrophobic SLA titanium discs has been shown to create superhydrophilic microstructures that enhance cellular osteogenesis compared to hydrophobic ones. ${ }^{33}$ However, extremely high surface energies may hinder cell motility and subsequent cell function. ${ }^{34}$ The optimal hydrophilicity of dental implants for best biological and clinical outcomes still remains unclear. Furthermore, DOPA-coating increases surface hydrophilicity, owing to its catechol and amine functional groups, ${ }^{35}$ and water contact angle decreases as a function of dopamine concentration, reflecting increased hydrophilicity. ${ }^{36}$ DOPA layer formation is a two-part process involving simultaneous adsorption and polymerization. During the polymerization process, a serious reaction can occur under alkaline conditions. For example, $o$ quinone was shown to be oxidized from the catechol group of DOPA and deposited on the surface through strong covalent interactions. ${ }^{37}$ In our previous study, we confirmed the surface chemistry of simple DOPA modification using XPS analysis, by revealing a nitrogen signal from the amine group. ${ }^{38}$ Non- 
specific interactions, including electrostatic charge and hydrophobic interactions, between the cell membrane and the substrate surface can cause cell adhesion. Hydrophobic surfaces have been reported as unfavorable for pre-adsorbed proteins adhesion and subsequent attachment. ${ }^{32}$ Moreover, physicochemical changes upon DOPA coating, which include surface roughness, wettability, and functional group changes, can all influence subsequent cell spreading and adhesion properties.

\subsection{DOPA-coating promotes adhesion, proliferation, and differentiation of BM-MSCs on SLA titanium discs}

BM-MSCs play a key role in bone regeneration around orthopedic implants or other bone tissue-engineered scaffolds, owing to their osteogenic differentiation potential. Cellular adhesion of BM-MSCs to the substrate is the initial stage of cellular migration and proliferation; therefore, direct and firm adhesion is important for proper implant osseointegration. Furthermore, the initial adhesion step is a prerequisite for osteogenic differentiation of MSCs into mature osteoblasts, responsible for bone formation on the bone-implant interface. ${ }^{39}$ We evaluated the influence of DOPA-coating on cellular adhesion of BM-MSCs, using cytoskeletal F-actin staining. We noted that cells exhibited a flatter and larger morphology on SLA-DOPA than on SLA alone at 1 and $3 \mathrm{~h}$ (Fig. 2a). Quantitative analysis showed that DOPA-coating enhanced cellular adhesion at 1,3 , and $24 \mathrm{~h}(P<$ 0.05) (Fig. 2b). This is consistent with previous studies that showed DOPA-coating increases cellular adhesion on modifiedsubstrates. ${ }^{28,40}$ Although it remains unclear how DOPA-coating improves cellular adhesion, reports have suggested that the increased surface energy is responsible. ${ }^{\mathbf{4 1 , 4 2}}$ Furthermore, several studies have postulated that cell behavior is regulated by serum proteins immobilized on DOPA-modified substrates, ${ }^{\mathbf{4 2}}$ or that attachment is influenced by cellular integrins. ${ }^{43}$ The results of our adhesion analysis indicate that DOPA-coating enhances cellular adhesion of BM-MSCs, which favors osteogenic differentiation during bone remodeling.

DOPA significantly increased BM-MSC proliferation at days 1 and 3, compared with SLA alone $(P<0.05)$, but not at day 7 (Fig. 2c). Previous reports have shown enhanced cellular proliferation on synthetic pDA-modified polymer substances. ${ }^{\mathbf{4 4 , 4 5}}$ ALP is an important marker of early osteoblastic differentiation. We noted DOPA coating significantly enhanced ALP activity of BM-MSCs compared with control cells at day 7 ( $P$ $<0.001$ ) (Fig. 2d), suggesting that DOPA-coating enhances osteoblastic differentiation. Similarly, Yu et al. showed that pDA-coating enhanced ALP activity of MC3T3-E1 cells on titanium at day 3. Our quantitative analysis of Alizarin Red S staining at 21 days indicates that DOPA-coating promotes osteogenic differentiation (Fig. 2e). In line with these observations, coating with dopamine has also been shown to promote osteogenic differentiation of MSCs on polymer fibers, accelerating human adipose-derived stem cells towards osteogenic differentiation. ${ }^{16}$ In our study, we used an osteogenic differentiation medium to induce osteogenic differentiation. We observed an enhanced effect of cells on DOPA-modified titanium discs in ALP and our quantitative calcium deposition assays indicated that MSCs exhibited a differentiated osteoblastic phenotype compared with cells on the untreated SLA surface. Previous studies have used osteogenic differentiation medium and proliferation medium simultaneously to analysis ALP activity, with cells treated in proliferation medium used as the negative control. ${ }^{31}$ Interestingly, no remarkable differences in ALP activity were observed for MG63 cells treated in proliferation medium between zirconia ceramics and DOPA-modified $\mathrm{ZrO}_{2}$ groups. ${ }^{26}$ MSCs grown on polydopamine-coated poly (Llactide) fibers have shown higher differentiation of ALP activity and osteogenic genes under the osteogenic conditions. ${ }^{46}$ These conflicting results of ALP and osteogenic activity could be attributed to the difference between cells, cell numbers, the level of protein expression and osteogenic conditions.

\subsection{Comparison of mRNA expression profiles of BM-MSCs cultured on titanium discs with or without DOPA coating}

We next used microarrays to compare mRNA expression profiles and identify key factors that control osteogenic differentiation of BM-MSCs cultured on SLA and SLA-DOPA discs for 7 days. We performed gene ontology (GO) enrichment analysis to determine differentially expressed genes during day seven, which is when MSCs on the DOPA-modified SLA surfaces exhibited a more differentiated phenotype compared with cells on the untreated SLA surface. Fig. 3a shows the differential gene expression was considered significant if the fold change was $>1.1$ or $<0.9$ and $P<0.05$. We focused on top $23 \mathrm{GO}$ functions and the vertical axis is the significant GO functions and the horizontal axis is the enrichment factor (enrichment factor > 1.5) in Fig. 3a. These GO functions were related to focal adhesion, cell-substance junction, RNA binding and other important relevant biological processes. Among these top $23 \mathrm{GO}$ functions, we focused on focal adhesion genes (Fig. 3b), which not only play an important role in cellular adhesion, but also behave as a key transducer of anchorage to the cell membrane, transferring information from the extracellular matrix (ECM). Fig. $3 \mathrm{~b}$ is the differential genes that participated in the GO function of focal adhesion, which is a key transducer of anchorage to the cell membrane, transferring information from the extracellular matrix (ECM). The heat map of Fig. 3b showed the differential gene in color gradation. Each ECM protein contains characteristic motifs, which are recognized by different integrins. Ligand-occupied integrins cluster and recruit other cytoskeletal proteins to stimulate formation of focal adhesions, establishing a connection between the cell and ECM. ${ }^{47}$ Proper cell-ECM attachment and formation of focal adhesions at the substrate interface play an important role in osteogenic differentiation and osteoneogenesis. ${ }^{48}$ Several studies have suggested that signaling pathways involving stimulation of mitogen-activated protein kinase (MAPK) and focal adhesion kinase (FAK) mediated by monomeric small G proteins, such as members of Rac, Cdc42 and Rho, are important transduction elements. Upregulation of gene expression related to the FAK-extracellular signal-regulated kinase (ERK)MAPK signaling pathway promotes MSC osteogenic 
a
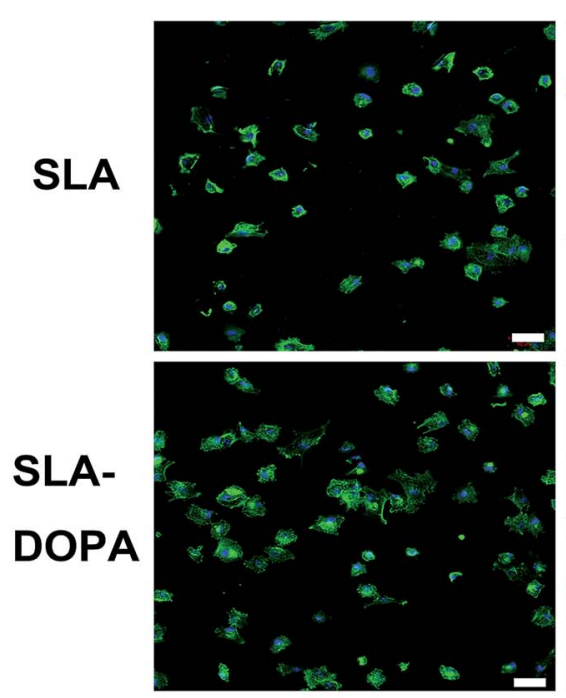

b
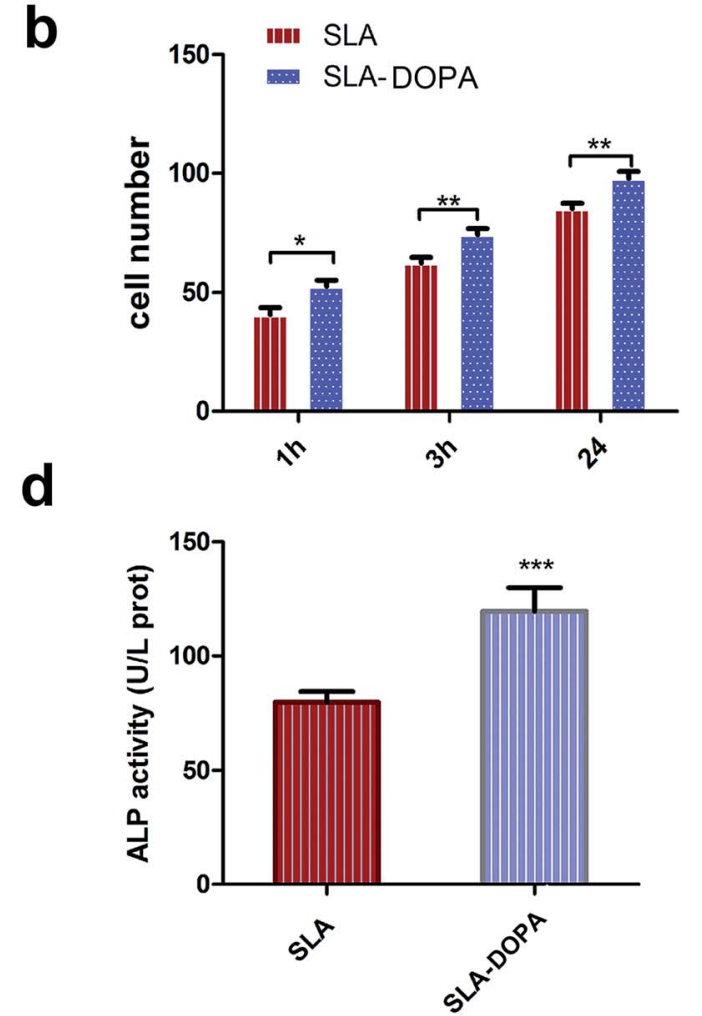

$3 h$
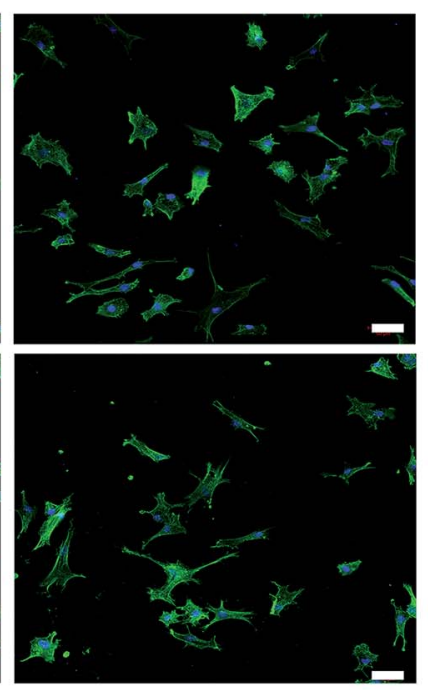

24h
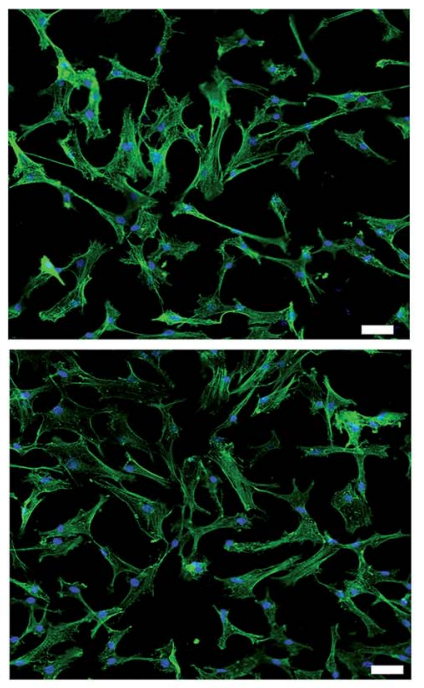

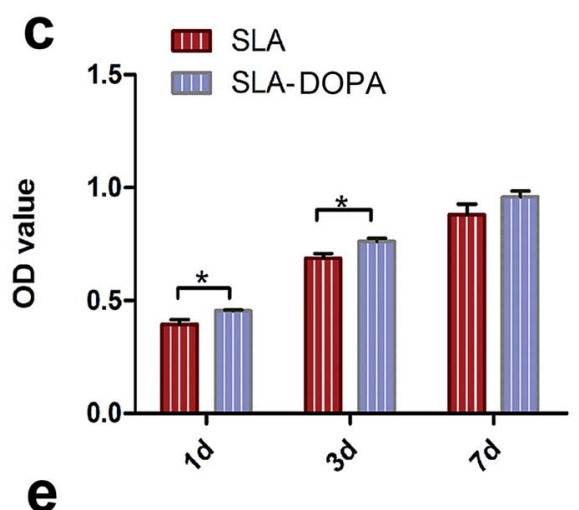

e

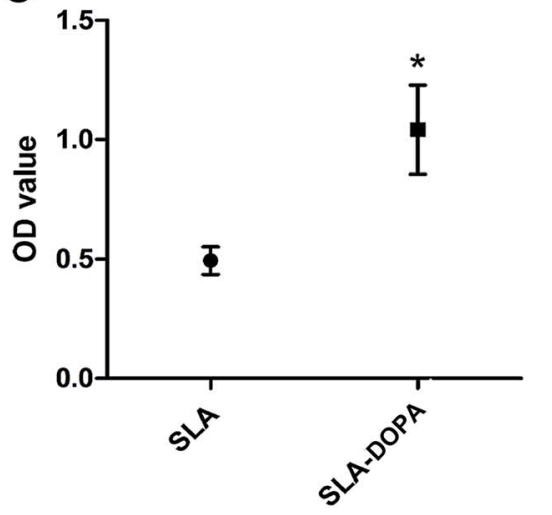

Fig. 2 Effects of SLA-DOPA coated titanium on the cellular responses of human bone marrow-derived mesenchymal stem cells (BM-MSCs) in vitro. (a) BM-MSC adhesion to SLA and SLA-DOPA 1, 3 and $24 \mathrm{~h}$ after seeding via FITC-conjugated phalloidin (green signal) and 4',6-diamidino-2phenylindole dihydrochloride (DAPI) staining (blue signal). Cells are more widely spread on SLA-DOPA at 1 and $3 \mathrm{~h}$. Scale bar $=50 \mu \mathrm{m}$. (b) DAPI staining of cell attachment; more pronounced cell adhesion is observed on SLA-DOPA at 1, 3 and $24 \mathrm{~h}$. (c) Cell counting kit- 8 assays of cell proliferation on day 1, day 3 and day 7. (d) Cellular alkaline phosphatase activity assay on day 7. (e) Quantification of cellular calcium mineralization on day 21. Error bars are mean $\pm \mathrm{SD}, n=3 ; * P<0.05 ; * * P<0.01 ; * * P<0.001$ vs. SLA.

differentiation. ${ }^{49,50}$ Using real-time PCR, we verified our microarray results. We revealed upregulation of SDC1, DPP4 and NF2 mRNA expression in BM-MSCs on SLA-DOPA discs compared with SLA alone (Fig. 3c). Cooperation between SDC1 and specific integrins plays a major role in matrix remodeling, matrix metalloproteinase secretion, cell adhesion, migration and cytoskeletal rearrangement. ${ }^{51}$ DPP4 has previously been reported to increase cell adhesion through the regulation of $\mathrm{E}$ cadherin, MMPs and TIMPs. ${ }^{52}$ NF-2 and its product merlin (moesin, ezrin, and radixin-like protein) are thought to be 
a

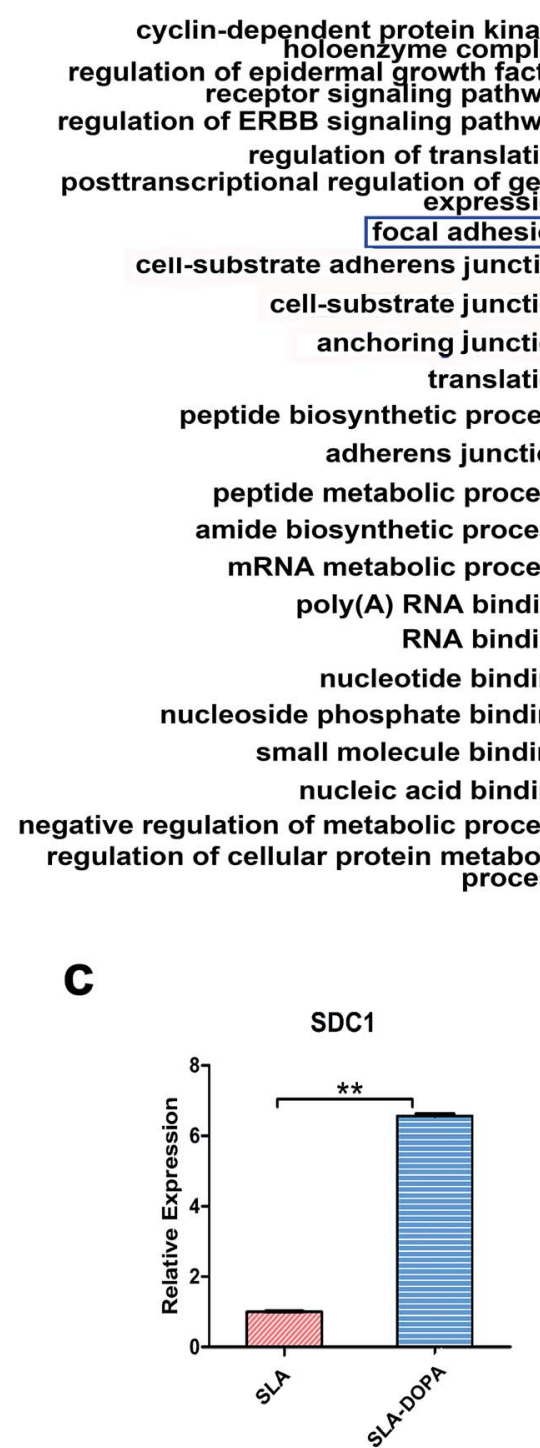

cyclin-dependent protein kinase

-|||||||||||||||||||||||||||||||||||||||||||||| $\mid$

$-||||||||||||||||||||||||||||||$
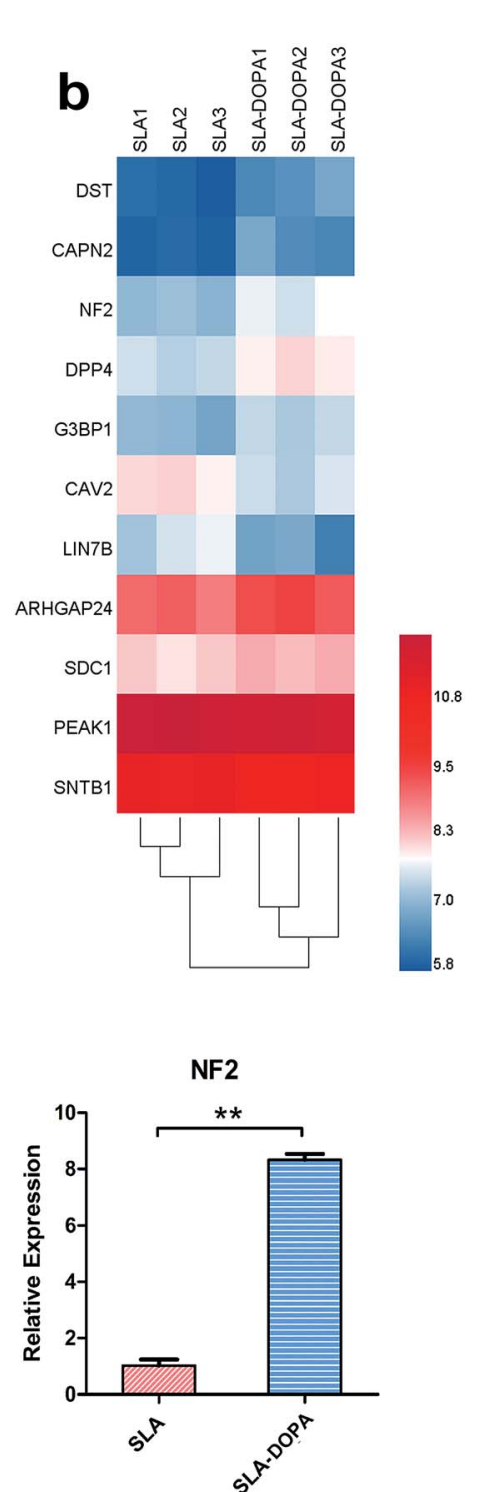

Fig. 3 DOPA modified SLA titanium influences gene expression in BM-MSCs. (a) Gene ontology pathway analysis for BM-MSCs on SLA and SLADOPA. Differential gene expression was considered significant if the fold change was $>1.1$ or $<0.9$ and $P<0.05$. The vertical axis displays the significant GO functions and the horizontal axis is the enrichment factor (enrichment factor $>1.5$ ). (b) Heat maps of differentially expressed genes for focal adhesion. Red represents high expression; blue represents low expression. (c) Expression levels of SDC1, DPP4 and NF2 mRNA in BMMSCs cultured on different surfaces after incubation for 7 days. Date are shown as mean $\pm \mathrm{SD} . * P<0.05$; **P<0.01 vs. SLA.

associated with the plasma membrane and cytoskeleton, which affect cell motility and signal transduction. ${ }^{53,54}$ In our study, we observed an up-regulation of genes, namely those involved in focal adhesion, in BM-MSCs seeded onto DOPA-coated surfaces. This mechanism is consistent with our observations of increased cellular adhesion and osteoblastic differentiation of BM-MSCs seeded onto DOPA-modified surfaces.

\section{Conclusions}

We characterized the effects of a simple polymerized DOPA film on titanium surfaces. SLA-DOPA titanium discs enhanced BMMSC adhesion, spreading, proliferation and differentiation, and upregulated expression of genes involved in focal adhesion in vitro. Therefore, our results suggest that surface modification with simple DOPA may provide a promising strategy to enhance new bone formation and remodeling around the implants.

\section{Conflicts of interest}

All authors declare that there are no conflicts of interest.

\section{Acknowledgements}

This work was supported by grants from the National Key R\&D Program of China (No. 2016YFC1102705), the National Nature Science Foundation of China (No. 81272966) and the Science 
Foundation of Peking University School and Hospital of Stomatology (PKUSS) (No. 20150106). The authors are grateful to Wego Jericom Biomaterials Co., Weihai, China, for kindly providing titanium samples. We thank Jishu Yin and Xinchang Li for technical assistance. The authors thank the Central Laboratory of PKUSS for offering facilities for this study.

\section{References}

1 P. I. Branemark, J. Prosthet. Dent., 1983, 50, 399-410.

2 J. M. Gomez-Vega, E. Saiz and A. P. Tomsia, J. Biomed. Mater. Res., 1999, 46, 549-559.

3 J. A. Bishop, A. A. Palanca, M. J. Bellino and D. W. Lowenberg, J. Am. Acad. Orthop. Surg., 2012, 20, 273282.

4 J. Raphel, M. Holodniy, S. B. Goodman and S. C. Heilshorn, Biomaterials, 2016, 84, 301-314.

5 S. Sisakhtnezhad, E. Alimoradi and H. Akrami, Eur. J. Cell Biol., 2017, 96, 13-33.

6 A. Chug, S. Shukla, L. Mahesh and S. Jadwani, J. Oral. Maxillofac. Surg. Med. Pathol., 2013, 25, 1-4.

7 A. I. Caplan, J. Pathol., 2009, 217, 318-324.

8 L. Feller, Y. Jadwat, R. A. Khammissa, R. Meyerov and I. Schechter, BioMed Res. Int., 2015, 2015, 171945.

9 T. K. Kovach, A. S. Dighe, P. I. Lobo and Q. Cui, J. Immunol. Res., 2015, 2015, 752510.

10 Q. Wang, H. Cheng, H. Peng, H. Zhou, P. Y. Li and R. Langer, Adv. Drug Delivery Rev., 2015, 91, 125-140.

11 G. Cheng, Z. Davoudi, X. Xing, X. Yu, X. Cheng, Z. Li, H. Deng and Q. Wang, ACS Biomater. Sci. Eng., 2018, 4, 2704-2715.

12 Y. Wen, S. Xun, M. Haoye, S. Baichuan, C. Peng, L. Xuejian, Z. Kaihong, Y. Xuan, P. Jiang and L. Shibi, Biomater. Sci., 2017, 5, 1690-1698.

13 J. Ng, K. Spiller, J. Bernhard and G. Vunjak-Novakovic, Tissue Eng., Part B, 2017, 23, 480-493.

14 Y. Liu, J. Lim and S.-H. Teoh, Biotechnol. Adv., 2013, 31, 688705.

15 L. P. Zhu, J. H. Jiang, B. K. Zhu and Y. Y. Xu, Colloids Surf., B, 2011, 86, 111-118.

16 K. H. Park, J. Y. Koak, S. K. Kim, C. H. Han and S. J. Heo, Int. J. Oral Maxillofac. Implant., 2013, 28, 57-66.

17 Y. Tian, Y. Cao, Y. Wang, W. Yang and J. Feng, Adv. Mater., 2013, 25, 2980-2983.

18 C. Y. Chien, T. Y. Liu, W. H. Kuo, M. J. Wang and W. B. Tsai, J. Biomed. Mater. Res., Part A, 2013, 101, 740-747.

19 H. S. Yang, J. Park, W. G. La, H. K. Jang, M. Lee and B. S. Kim, Tissue Eng., Part C, 2012, 18, 245-251.

20 H. Lee, B. P. Lee and P. B. Messersmith, Nature, 2007, 448, 338-341.

21 H. Lee, S. M. Dellatore, W. M. Miller and P. B. Messersmith, Science, 2007, 318, 426-430.

22 H. G. Silverman and F. F. Roberto, Mar. Biotechnol., 2007, 9, 661-681.

23 Y. T. Sul, Biomaterials, 2003, 24, 3893-3907.

24 L. Le Guehennec, A. Soueidan, P. Layrolle and Y. Amouriq, Dent. Mater., 2007, 23, 844-854.
25 D. R. Dreyer, D. J. Miller, B. D. Freeman, D. R. Paul and C. W. Bielawski, Langmuir, 2012, 28, 6428-6435.

26 Y. T. Liu, T. M. Lee and T. S. Lui, Colloids Surf., B, 2013, 106, 37-45.

27 X. Yu, J. Walsh and M. Wei, RSC Adv., 2013, 4, 7185-7192.

28 S. H. Kim, J. K. Park, J. H. Hong, H. S. Jung, K. S. Hong, J. H. Lee, K. B. Park, S. K. Choi and Y. K. Seo, J. Biomater. Appl., 2012, 27, 143-152.

29 H. Peng, X. Liu, R. Wang, F. Jia, L. Dong and Q. Wang, J. Mater. Chem. B, 2014, 2, 6435-6461.

30 E. C. Combe, B. A. Owen and J. S. Hodges, Dent. Mater., 2004, 20, 262-268.

31 M. A. Surmeneva, C. Kleinhans, G. Vacun, P. J. Kluger, V. Schonhaar, M. Muller, S. B. Hein, A. Wittmar, M. Ulbricht, O. Prymak, C. Oehr and R. A. Surmenev, Colloids Surf., B, 2015, 135, 386-393.

32 R. A. Gittens, L. Scheideler, F. Rupp, S. L. Hyzy, J. GeisGerstorfer, Z. Schwartz and B. D. Boyan, Acta Biomater., 2014, 10, 2907-2918.

33 I. Wall, N. Donos, K. Carlqvist, F. Jones and P. Brett, Bone, 2009, 45, 17-26.

34 S. Ernst, S. Stubinger, P. Schupbach, M. Sidler, K. Klein, S. J. Ferguson and B. von Rechenberg, Clin. Oral Implant. Res., 2015, 26, 898-908.

35 H. Lee, J. Rho and P. B. Messersmith, Adv. Mater., 2009, 21, 431-434.

36 Z.-Y. Xi, Y.-Y. Xu, L.-P. Zhu, Y. Wang and B.-K. Zhu, J. Membr. Sci., 2009, 327, 244-253.

37 S. Haemers, G. J. Koper and G. Frens, Biomacromolecules, 2003, 4, 632-640.

38 T. Ma, X. Y. Ge, K. Y. Hao, B. R. Zhang, X. Jiang, Y. Lin and Y. Zhang, Sci. Rep., 2017, 7, 17849.

39 K. C. Popat, L. Leoni, C. A. Grimes and T. A. Desai, Biomaterials, 2007, 28, 3188-3197.

40 N. Li, G. Chen, J. Liu, Y. Xia, H. Chen, H. Tang, F. Zhang and N. Gu, ACS Appl. Mater. Interfaces, 2014, 6, 17134-17143.

41 C. Ozcan and N. Hasirci, J. Biomater. Sci., Polym. Ed., 2007, 18, 759-773.

$42 \mathrm{~S} . \mathrm{H} . \mathrm{Ku}$, J. Ryu, S. K. Hong, H. Lee and C. B. Park, Biomaterials, 2010, 31, 2535-2541.

43 J. L. Wang, K. F. Ren, H. Chang, F. Jia, B. C. Li, Y. Ji and J. Ji, Macromol. Biosci., 2013, 13, 483-493.

44 Y. J. Lee, J. H. Lee, H. J. Cho, H. K. Kim, T. R. Yoon and H. Shin, Biomaterials, 2013, 34, 5059-5069.

45 H. J. Cho, S. K. Perikamana, J. H. Lee, J. Lee, K. M. Lee, C. S. Shin and H. Shin, ACS Appl. Mater. Interfaces, 2014, 6, 11225-11235.

46 N. G. Rim, S. J. Kim, Y. M. Shin, I. Jun, D. W. Lim, J. H. Park and H. Shin, Colloids Surf., B, 2012, 91, 189-197.

47 H. J. Anderson, J. K. Sahoo, R. V. Ulijn and M. J. Dalby, Front. Bioeng. Biotechnol., 2016, 4, 38.

48 M. J. Biggs and M. J. Dalby, Proc. Inst. Mech. Eng. H, 2010, 224, 1441-1453.

49 P. Tomakidi, S. Schulz, S. Proksch, W. Weber and T. Steinberg, Cell Tissue Res., 2014, 357, 515-526.

50 C. B. Khatiwala, P. D. Kim, S. R. Peyton and A. J. Putnam, J. Bone Miner. Res., 2009, 24, 886-898. 
51 X. Xian, S. Gopal and J. R. Couchman, Cell Tissue Res., 2010, 339, 31-46.

52 F. Kikkawa, H. Kajiyama, K. Shibata, K. Ino, S. Nomura and S. Mizutani, Biochim. Biophys. Acta, 2005, 1751, 45-51.

53 W. Li, J. Cooper, M. A. Karajannis and F. G. Giancotti, EMBO Rep., 2012, 13, 204-215.
54 N. R. Shah, I. Tancioni, K. K. Ward, C. Lawson, X. L. Chen, C. Jean, F. J. Sulzmaier, S. Uryu, N. L. Miller, D. C. Connolly and D. D. Schlaepfer, Gynecol. Oncol., 2014, 134, 104-111. 